\title{
The Center for HIV/AIDS Vaccine Immunology (CHAVI) multi-site quality assurance program for cryopreserved Human Peripheral Blood Mononuclear Cells
}

\author{
Marcella Sarzotti-Kelsoe ${ }^{\mathrm{a}, \mathrm{d}, \mathrm{g}, *, 1}$, Leila K. Needham ${ }^{\mathrm{a}, 1}$, Wes Rountree ${ }^{\mathrm{a}}$, John Bainbridge ${ }^{\mathrm{a}}$, \\ Clive M. Gray ${ }^{\mathrm{b}, 2}$, Susan A. Fiscus ${ }^{c}$, Guido Ferrari ${ }^{\mathrm{a}, \mathrm{d}}$, Wendy S. Stevens ${ }^{\text {e,f }}$, Susan L. Stager ${ }^{\mathrm{a}, \mathrm{d}}$, \\ Whitney Binz ${ }^{a}$, Raul Louzao ${ }^{a}$, Kristy O. Long ${ }^{d}$, Pauline Mokgotho ${ }^{b}$, Niranjini Moodley ${ }^{\text {b,f }}$, \\ Melanie Mackay ${ }^{\text {b,f }}$, Melissa Kerkau ${ }^{c}$, Takesha McMillion ${ }^{\text {c }}$, Jennifer Kirchherr ${ }^{\text {a }}$, \\ Kelly A. Soderberg ${ }^{a}$, Barton F. Haynes ${ }^{\text {a }}$, Thomas N. Denny ${ }^{\text {a }}$ \\ a Duke Human Vaccine Institute, Duke University Medical Center, Durham, NC, USA \\ b AIDS Virus Research Unit, National Institute for Communicable Diseases, Johannesburg, South Africa \\ c Department of Microbiology and Immunology, University of North Carolina at Chapel Hill, Chapel Hill, NC, USA \\ d Department of Surgery, Duke University Medical Center, Durham, NC, USA \\ e Department of Molecular Medicine and Haematology, Faculty of Health Sciences, University of the Witwatersrand and the National Health Laboratory Services, \\ Johannesburg, South Africa \\ ${ }^{\mathrm{f}}$ Clinical Laboratory Services (CLS), Johannesburg, South Africa \\ g Department of Immunology, Duke University Medical Center, Durham, NC, USA
}

\section{A R T I C L E I N F O}

\section{Article history:}

Received 9 July 2013

Received in revised form 23 May 2014

Accepted 28 May 2014

Available online $\mathrm{xxxx}$

\section{Keywords:}

Cryopreservation

Vaccine

Human clinical trials

PBMC

HIV

Biorepository

\begin{abstract}
A B S T R A C T
The Center for HIV/AIDS Vaccine Immunology (CHAVI) consortium was established to determine the host and virus factors associated with HIV transmission, infection and containment of virus replication, with the goal of advancing the development of an HIV protective vaccine. Studies to meet this goal required the use of cryopreserved Peripheral Blood Mononuclear Cell (PBMC) specimens, and therefore it was imperative that a quality assurance (QA) oversight program be developed to monitor PBMC samples obtained from study participants at multiple international sites. Nine site-affiliated laboratories in Africa and the USA collected and processed PBMCs, and cryopreserved PBMC were shipped to CHAVI repositories in Africa and the USA for long-term storage. A three-stage program was designed, based on Good Clinical Laboratory Practices (GCLP), to monitor PBMC integrity at each step of this process. The first stage evaluated the integrity of fresh PBMCs for initial viability, overall yield, and processing time at the site-affiliated laboratories (Stage 1); for the second stage, the repositories determined post-thaw viability and cell recovery of cryopreserved PBMC, received from the site-affiliated laboratories (Stage 2); the third stage assessed the long-term specimen storage at each repository (Stage 3). Overall, the CHAVI PBMC QA oversight program results highlight the relative importance of each of these stages
\end{abstract}

\footnotetext{
* Corresponding author at: Duke University Medical Center, 2812 Erwin Rd., Suite 301, Erwin Terrace II, Durham, NC 27705, USA. Tel.: + 1919 684 6373; fax: + 1919 6817766.

E-mail address: marcella.sarzottikelsoe@dm.duke.edu (M. Sarzotti-Kelsoe).

1 Both first authors.

${ }^{2}$ Current address: Division of Immunology, Institute of Infectious Disease and Molecular Medicine, University of Cape Town, Faculty of Health Sciences, Cape Town, South Africa.
} 
to the ultimate goal of preserving specimen integrity from peripheral blood collection to long-term repository storage.

(c) 2014 Elsevier B.V. All rights reserved.

\section{Introduction}

The Center for HIV/AIDS Vaccine Immunology (CHAVI) sought to use Peripheral Blood Mononuclear Cell (PBMC) specimens obtained from study participants enrolled at multiple international sites to determine host immune responses during acute HIV-1-infection and to identify determinants of host resistance to HIV-1-infection. PBMCs were isolated and cryopreserved at nine international site-affiliated laboratories and shipped to the repositories for long-term storage and subsequent distribution to CHAVI specialized laboratories (i.e., end users). The need for high quality PBMC necessitated the development of a quality assurance (QA) program to monitor the quality of PBMC isolated and stored.

Here, we describe a comprehensive, Good Clinical Laboratory Practices - (GCLP) compliant CHAVI QA oversight program designed to monitor initial PBMC integrity upon isolation at the site-affiliated laboratories and post-thaw viability and recovery of cryopreserved PBMC at the repositories. This program also assessed the integrity of PBMCs held in long-term storage at the repositories. Acceptance criteria were pre-established for key quality indicators, and corrective actions were implemented in the event of QC failure. The program included site remediation to improve the quality of the cryopreserved PBMC.

In developing the program, multiple criteria were monitored to ensure reliable results in functional and phenotypic assays when the PBMCs were thawed at endpoint laboratories. Quality control $(\mathrm{QC})$ indicators of initial PBMC integrity include viability, PBMC yield/mL of whole blood, and the time elapsed between blood collection and cryopreservation (Bull et al., 2007; Dyer et al., 2007; Kierstead et al., 2007; Olemukan et al., 2010). Previous studies have highlighted the importance of obtaining post-thaw PBMC viabilities of $\geq 70 \%$ since the results of functional and phenotypic studies are strictly dependent on the viability of the cryopreserved PBMCs (Cox et al., 2005; Kreher et al., 2003; Reimann et al., 2000; Sleasman et al., 1997; Weinberg et al., 2000, 2009, 2010). It is also essential that adequate numbers of cells are recovered from the freezing process to perform most PBMC functional assays. Therefore, the dual parameters of post-thaw viability and recovery of cryopreserved PBMCs must be monitored (Aziz et al., 2013; Weinberg et al., 2007). Additional studies also indicated the importance of cryopreserving PBMCs within $8 \mathrm{~h}$ of blood collection since longer processing times adversely affect the performance of $\mathrm{T}$ cells in immunological assays (Bull et al., 2007).

\section{Materials and methods}

\subsection{Study participants}

The CHAVI prospective, observational Clinical Study Protocols were reviewed and approved by the Institutional Review Board of Duke University Medical Center and by the responsible
Institutional Review Boards or Ethical Committees in the USA, Malawi, South Africa, Tanzania, and Uganda. All study participants provided written informed consent for the collection, testing and subsequent analysis of the study samples.

The longitudinal multicenter CHAVI studies enrolled participants that were HIV-infected [acutely $-(n=376)$, chronically $-(n=420)$, undefined $(n=184)]$ and uninfected [with autoimmune disease $(\mathrm{n}=322)$ or without $(\mathrm{n}=348)$ ], on multiple visits.

Site-affiliated laboratories were established at the Aaron Diamond AIDS Research Center, New York, New York, USA; the Aurum Institute for Health Research, Klerksdorp, South Africa; the Centre for the AIDS Programme of Research in South Africa, University of KwaZulu-Natal, Durban, South Africa; Clinical Laboratory Services, National Health Laboratory Service/Wits Health Consortium of the University of the Witwatersrand, Johannesburg, South Africa; Duke University, Durham, North Carolina, USA; the Johns Hopkins University Research Project (JHUR Project), Malawi College of Medicine, Blantyre, Malawi; the Kilimanjaro Christian Medical Centre, Moshi, Tanzania; the Medical Research Council Programme on AIDS/Uganda Virus Research Institute, Entebbe, Uganda; and the University of North Carolina Project - Malawi (UNC Project), Lilongwe, Malawi.

Two CHAVI central repositories were established at the National Institute of Communicable Diseases in Johannesburg South Africa (repository 1), and at the University of North Carolina (UNC) in Chapel Hill, North Carolina, USA (repository 2), for long-term storage of PBMC specimens shipped from the site-affiliated laboratories. In order to avoid unnecessary shipments, a CHAVI repository was also established at Duke University, Durham, NC, USA (repository 3) for long-term storage of PBMC specimens prepared by the Duke University site-affiliated laboratory.

\subsection{PBMC QA program}

The CHAVI QA oversight program was designed to monitor PBMC integrity at three stages of PBMC isolation and storage (Fig. 1).

\subsubsection{Stage $1-P B M C$ initial viability, yield, and processing time at the site-affiliated laboratories}

2.2.1.1. Training. To standardize PBMC isolation and cryopreservation, each site-affiliated laboratory operated in compliance with required standards for GCLP (Ezzelle et al., 2008; Sarzotti-Kelsoe et al., 2009; Stiles et al., 2003). Operators used identical CHAVI central standard operating procedures (SOPs) for the processing, labeling, transporting and storage of PBMC specimens. Staff from the site-affiliated laboratories participated in wet-laboratory training provided by the CHAVI repositories. Each trainee had to successfully participate in a PBMC processing qualification run before processing study participant PBMCs for CHAVI, and competency was 


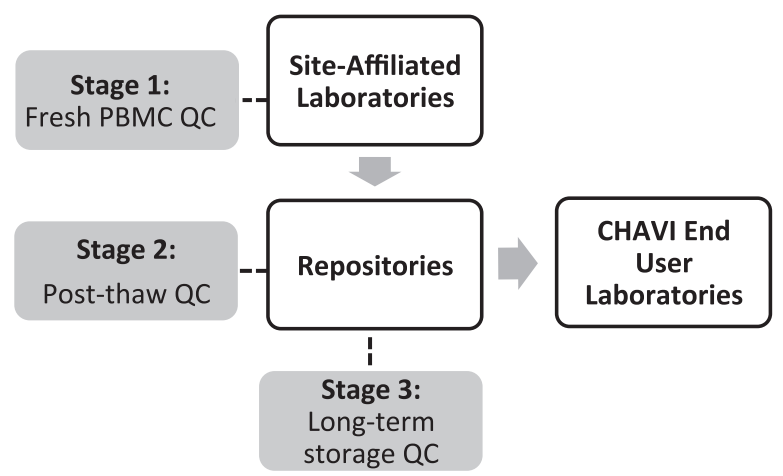

Fig. 1. Design of the CHAVI PBMC QA Oversight Program. This schematic illustrates the shipment of PBMCs from the site-affiliated laboratories to the repositories and to end user laboratories and the stages of the CHAVI PBMC QA oversight program: Stage 1 - PBMC initial viability, yield, and processing time at the site-affiliated laboratories; Stage 2 - cryopreserved PBMC viability and cell recovery after thawing at the repositories; and Stage 3 Sentinel Program for long-term storage of cryopreserved PBMCs at the repositories.

assessed annually thereafter. The CHAVI Central Quality Assurance Unit, located at Duke University, assessed the overall GCLP compliance of the program, by monitoring that all processes were planned, performed, monitored, recorded, and reported in a reliable and consistent manner. The site-affiliated laboratories and the repositories were audited annually by the sponsor or the CHAVI Central Quality Assurance Unit for adherence to GCLP standards.

2.2.1.2. PBMC processing. Blood was collected into acidcitrate-dextrose anticoagulant blood collection tubes and transported to each site-affiliated laboratory at room temperature. PBMCs were isolated from blood, following CHAVI Central SOPs, by centrifugation at $800 \times g$ for $30 \mathrm{~min}$ at room temperature, through Ficoll-paque Plus (GE Healthcare Life Sciences, Pittsburgh, PA) using Leucosep (Greiner Bio-One, North America, Monroe, NC) or Accuspin (Sigma-Aldrich, St. Louis, MO) tubes. The PBMC layer was harvested and washed three times with Hank's Balanced Salt Solution. PBMCs were cryopreserved at a concentration of $\approx 1 \times 10^{7}$ cells $/ \mathrm{mL}$ in cryopreservation solution (90\% fetal bovine serum (FBS), $10 \%$ dimethyl sulfoxide), using cell freezing containers (e.g., "Mr. Frosty" Nalgene Labware; StrataCooler, Stratagene; or CoolCell, BioCision, LLC) to control the rate of freezing overnight in a $-70 / 80{ }^{\circ} \mathrm{C}$ freezer. Although PBMC storage at $-70{ }^{\circ} \mathrm{C}$ for up to three weeks does not affect recovery and/or viability upon thawing (Disis et al., 2006; Bull et al., 2007), the site-affiliated laboratories were required by SOP, to transfer cryopreserved cells on the next working day (within $72 \mathrm{~h}$ ) from $-70{ }^{\circ} \mathrm{C}$ to storage in liquid nitrogen vapor at $\leq-140^{\circ}$. This process was performed to minimize risk and preserve specimen integrity. Cryopreserved PBMC specimens were temporarily stored in liquid nitrogen freezers at the site-affiliated laboratories and were subsequently shipped by cryoshipper to repositories 1 and 2 for long-term storage in vapor phase liquid nitrogen. At later dates, PBMC samples were shipped to CHAVI end users for specialized functional testing. Only PBMCs isolated at the Duke University site-affiliated laboratory were stored on-site at repository 3 .
Three quality control (QC) indicators were recorded and monitored for each PBMC specimen processed by the siteaffiliated laboratories: the percentage of viable PBMCs at the time of isolation (initial viability); the number of PBMC isolated per $\mathrm{mL}$ of blood (yield); the time elapsed between blood collection and initiation of cryopreservation (processing time). These three parameters had to meet established acceptance criteria. Acceptance criteria for PBMC initial viability, yield, and processing time results, established by the HIV Vaccine Trials Network (HVTN) (0.8-3.2 × $10^{6} \mathrm{PBMC} / \mathrm{mL}$ blood) (Ducar et al., 2014) and by other groups (Afonso et al., 2010) were initially used and adjusted over a pilot study, to the CHAVI population. The CHAVI acceptance criteria, used in all of the studies described in this manuscript, for PBMC percent viability after isolation were established as $\geq 95 \%$ and for the PBMC yield as $0.7-3.0 \times 10^{6} \mathrm{PBMC} / \mathrm{mL}$ blood. The processing time acceptance criteria were set at $\leq 8 \mathrm{~h}$ based upon previous studies (Bull et al., 2007; Kierstead et al., 2007). Once a site-affiliated laboratory was activated to participate in CHAVI PBMC processing, PBMC viability, PBMC yield, and PBMC processing time were monitored on monthly trend-lines by the manager of the CHAVI central repository 1 . For values outside the pre-set acceptance criteria, the repository manager worked with the site-affiliated laboratories to identify the sources and magnitude of the difficulties and to develop a corrective action plan, including retraining by the repositories and site visits where necessary.

2.2.1.3. PBMC shipment from the site-affiliated laboratories to the repositories. Approximately half of the cryovials from each PBMC donor were shipped separately by the site-affiliated laboratories to each central repository (repositories 1 and 2) to reduce the risk of loss due to shipment, equipment, or facility failures. The cryovials were shipped in MVE IATA approved cryoshippers (Chart Biomedical Division, Chart Industries, Inc., Ball Ground, GA) to maintain the cells at $\leq-150{ }^{\circ} \mathrm{C}$ (Smith et al., 2007; Weinberg et al, 2010). Cryoshippers were equipped with continuously recording temperature dataloggers. QC procedures verified the integrity of the cryoshippers prior to each shipment. PBMCs were stored at the CHAVI repositories in vapor phase liquid nitrogen freezers at $\leq-140{ }^{\circ} \mathrm{C}$. A CHAVI standardization study indicated that, after removal from liquid nitrogen vapor, cryopreserved specimens warmed within $15 \mathrm{~s}$ to the critical cryogenic temperature of $-130{ }^{\circ} \mathrm{C}$. Thus, a central CHAVI SOPs was developed and mandated that all cryopreserved PBMCs be handled in a liquid nitrogen-vapor environment using temperature-monitored transfer pans or CryoCarts (Chart Industries, Inc.). Specimens were tracked by linking NIAID/DAIDS Laboratory Data Management System (LDMS) units (provided by Frontier Science and Technology Research Foundation) to the central database, Atlas (managed by the Statistical Center for HIV/AIDS Research and Prevention). Each study participant had a unique Participant Identification Number (PTID), and each specimen aliquot had a unique global specimen identification number that was linked to the participant and visit.

\subsubsection{Stage $2-$ Cryopreserved PBMC viability and cell recovery after thawing at the repositories}

To assess the integrity of the PBMCs after cryopreservation, handling and shipment to the repositories, PBMCs from each site-affiliated laboratory were sampled by the CHAVI 
specimen manager and analyzed quarterly by the central repositories for post-thaw viability and total cell recovery. HIV-seronegative specimens were primarily selected and thawed for QC assessment; PBMC specimens isolated from chronically HIV-infected participants were selected if no HIV-negative specimens were available. If possible, at least two PBMC specimen vials were selected quarterly for QC assessment. No PBMC preparations were thawed for assessment from two of the nine site-affiliated laboratories since no eligible study participants were enrolled.

2.2.2.1. PBMC thawing. PBMC were rapidly thawed, following a CHAVI central SOP, in a $37^{\circ} \mathrm{C}$ water bath and slowly diluted with an equivalent volume of pre-warmed $\left(37^{\circ} \mathrm{C}\right.$ ) (Disis et al., 2006; Ramachandran et al., 2012) complete medium (RPMI 1640, 10\% FBS and antibiotics) plus 50 Units $/ \mathrm{mL}$ Benzonase ${ }^{\circledR}$ Nuclease (Novagen, EMD Millipore Division, Merck KGaA, Darmstadt, Germany) (Smith et al., 2001). Cell counts and percent viability were determined on the day of the thaw (Day 0 ) and after overnight rest (Day 1) in complete medium. Cell counts and viability were determined with a hemacytometer, a Vi-Cell instrument (Beckman Coulter) using trypan blue exclusion, or by a Guava Personal Cell Analysis (PCA) instrument (Millipore Technologies), using Guava ViaCount reagent. Instruments were qualified by comparison with hemacytometer counts. Since a set of experiments performed by the central repository 2 , comparing Vi-Cell and hemacytometer counts indicated that red blood cells (RBCs) contributed to the Vi-Cell counts, RBCs were lysed using Versalyse (Beckman Coulter) prior to counting PBMC on the Vi-Cell.

Total cell recovery was calculated as the total cells recovered after thaw divided by the original vial cell content, $\times 100$. Total cell recovery was used as one $\mathrm{QC}$ indicator to monitor the number of PBMCs that the site-affiliated laboratories actually dispensed in the vial at cryopreservation. Viability for each cryovial was determined as well upon thawing, but analyzed as a separate QC value. Both QC measures, total cell recovery and the fraction of viable cells after thaw, are necessary to effectively evaluate site performance. Based on post-thaw viability and total cell recovery results initially established from PBMC thawed for ELISpot analysis (Dr. Ferrari, personal communication), the acceptance criteria for post-thaw viability was defined as $\geq 80 \%$, and for the post-thaw total cell recovery was defined as 55$120 \%$. If a QC result did not meet the preset acceptance criteria, the sample was recounted to verify the results. If the out of criteria result was obtained with an automated cell-counting instrument, the recount was performed using a hemacytometer and trypan blue. Inter-operator hemacytometer counting comparisons were performed quarterly, and repository operators were assessed annually for control sample thawing competency.

\subsubsection{Stage 3 - Sentinel Program for long-term storage of} cryopreserved PBMCs at the repositories

Stage 3 of the PBMC QA Program was designed to monitor the long-term storage of cryopreserved PBMC at the CHAVI repositories. To this end, a Sentinel Program was designed using a large number of identical cryovials (control samples), containing cryopreserved PBMC at a concentration of $\approx 1 \times 10^{7}$ cells $/ \mathrm{mL}$ from a single, HIV-negative leukapheresis participant (see Garcia et al. in this issue), prepared by the Immunology Quality Assessment Center (IQAC). These sentinel control samples were shipped to all repositories for storage in each liquid nitrogen freezer containing CHAVI cryopreserved PBMCs. In these freezers, the sentinel control samples were exposed to the same storage conditions and potential temperature excursions as the CHAVI cryopreserved PBMCs. Each quarter, all repositories thawed three control sample vials from each freezer(s) and determined the post-thaw percent viability and total cell recovery. As an independent third party verification, all repositories sent three additional control sample vials by cryoshipper to the IQAC each quarter for assessment. The acceptance criteria for post-thaw control sample viability and total cell recovery were set during the first quarter when the three repositories and the IQAC each thawed six control sample vials. The consensus mean and SD were used to set acceptance criteria specific for the single control sample batch used during the entire QC cycle; acceptable post-thaw viability was defined as $\geq 92.9 \%$ and post-thaw recovery for the control samples as $57.7-120.7 \%$. If individual control samples QC values were outside the acceptance criteria, the repository or IQAC manager initiated investigative/ corrective action steps to ensure that cell-counting procedures were accurate and that the PBMC integrity was maintained.

\subsection{Statistical analysis}

Statistical comparisons were made using regression and mixed effects models in SAS Version 9.2. These models were run using PROC MIXED to perform equivalence tests, using a simple $1-\alpha$ confidence interval, based on the population means. An example of the null hypothesis based on Stage 1 is that the absolute value of the site-affiliated laboratory mean minus the consensus mean is greater than the equivalence margin. The alternative hypothesis is that the absolute value of the site-affiliated laboratory mean minus the consensus mean is less than or equal to the equivalence margin. The alpha level for all tests was set at 0.05, and a significant p-value $(<0.05)$ indicates that the null hypothesis is rejected and equivalence (based on the equivalence margin) can be claimed. No adjustment to the significance level was made for these analyses. The equivalence margins are as follows: for initial and post-thaw viability, it is 5 percentage points; for PBMC yield, it is $0.5 \times 10^{6}$ cells $/ \mathrm{mL}$ blood; for PBMC processing time, it is $2 \mathrm{~h}$; and for post-thaw recovery, it is 15\%. The Stage 1 analysis compared each site-affiliated laboratory's difference from the consensus mean, as well as pairwise HIV-infection cohort comparisons. Stage 2 comparisons were based on group determinations based on non-overlapping $95 \%$ confidence intervals $(\mathrm{CI})$ of the mean post-thaw QC values for each site-affiliated laboratory. These comparisons were made with estimates from mixed effects models. Stage 3 comparisons were made using a regression model.

\section{Results}

3.1. Stage 1 - PBMC initial viability, yield, and processing time at the site-affiliated laboratories

Data were collected from the nine site-affiliated laboratories processing CHAVI PBMC specimens over five years. We analyzed 
PBMC values from CHAVI cohorts of acutely HIV-infected ( $\mathrm{n}=$ 1125), chronically HIV-infected $(n=626)$ and HIV-negative ( $\mathrm{n}=297$ ) participants and found that initial PBMC viabilities, yield, and processing time were equivalent (all p-values $<0.001$; data not shown) for all cohorts. Thus, the results in Table 1 are derived from combined data from HIV-positive and HIV-negative participants $(\mathrm{n}=8554)$. As shown in Table 1 , the mean PBMC initial viability, yield, and processing time for all site-affiliated laboratories were within the acceptance criteria.

Statistical comparisons were performed using mixed effects models in order to determine whether sites were equivalent to the consensus mean. The model-based mean viabilities at all site-affiliated laboratories were found equivalent to the consensus mean (all p-values $<0.001$ ). For PBMC yield, the model-based mean for 8/9 site-affiliated laboratories was equivalent to the consensus mean (one p-value $=0.857$, otherwise all p-values $<0.007$ ), and the processing times at $8 / 9$ site-affiliated laboratories were equivalent to the consensus mean (one p-value $=0.656$, otherwise all p-value $<0.02$ ). The higher PBMC yields observed at one site decreased after the site began to verify any out of criteria yield results obtained with the Vi-Cell automated counter, by using hemacytometer counts. Another site's processing time was consistently lower than the consensus mean value, which had no biological consequence on the PBMC specimen integrity as all sites were within the acceptance criteria of less than $8 \mathrm{~h}$.

\subsection{Stage $2-$ Cryopreserved PBMC viability and cell recovery after thawing at the repositories}

The three repositories evaluated the post-thaw viability and recovery of selected cryopreserved PBMCs from the site-affiliated laboratories. The post-thaw PBMC QC data collected from the seven site-affiliated laboratories over five years from 252 PBMC isolates are presented in Fig. 2. Sixtynine percent of the thawed PBMC specimens passed the post-thaw PBMC viability acceptance criteria (mean viability +/- SD: $80.1+/-20.3 \%)$. As shown in Fig. 2A, the mean post-thaw PBMC viability was within the acceptance criteria at four of the seven site-affiliated laboratories. Statistical comparisons, based on non-overlapping model-based 95\% CI of the mean post-thaw viability for each site-affiliated laboratory, indicated that site-affiliated laboratories 5,6 , and 7 differed from the other site-affiliated laboratories. These three site-affiliated laboratories exhibited mean post-thaw PBMC viability values that were below the acceptance criteria (76.5, 63.6, and $61.2 \%$, respectively).

With respect to the PBMC recovery QC results, $72.6 \%$ of the thawed PBMC isolates passed the acceptance criteria (mean $+/-$ SD: $77.2 \%+/-35.1 \%$ ). As shown in Fig. 2B, the mean PBMC recovery was within the acceptance criteria at each site-affiliated laboratory. The percentage of PBMC preparations that failed due to recoveries of $>120 \%$ decreased during the PBMC QA period, suggesting that counting errors and/or errors in seeding high numbers of cells/vial were more prevalent during the early period (data not shown).

In order to determine if the key post-thaw QC indicators for the PBMC isolated by the site-affiliated laboratories improved during the span of the PBMC QA Program, the quarterly mean post-thaw QC results obtained from PBMC isolated at each site-affiliated laboratory over time was examined (data not shown). Site-affiliated laboratories 6 and 7 had post-thaw viability values that were consistently below the acceptance criteria, although the viability appeared to improve over time.

The quarterly mean post-thaw PBMC total cell recovery results were within the acceptance criteria at each site-affiliated laboratory.

\subsection{Stage 3 - Sentinel Program for long-term storage of cryopreserved PBMCs at the repositories}

Stage 3 of the PBMC QA oversight program was designed to monitor the long-term storage of cryopreserved PBMCs at the CHAVI repositories, by assessing post-thaw viability and recovery of identical sentinel control samples stored in each liquid nitrogen freezer containing CHAVI cryopreserved PBMCs.

Two years of control sample thawing QC data $(n=406)$ from the three CHAVI repositories and the IQAC were analyzed statistically for comparisons between Day 0 and Day 1 mean post-thaw viability and total cell recovery results. There was no evidence that the Day 0 and Day 1 control sample viabilities were not equivalent $(p<0.001)$. Therefore, these results were combined for analysis. However, the Day 0 and Day 1 model-based mean control sample recoveries were not equivalent $(p=0.519)$, with a lower recovery on Day 1 . Therefore, the results for Day 1 are reported as PBMC would typically be rested for a day prior to assay use (Kierstead et al., 2007) In addition, the mean control sample post-thaw viability and recovery data were analyzed statistically to test whether the shipment of the control samples between the repositories and the IQAC affected sample integrity. There was no evidence that the control sample post-thaw viabilities or recoveries were not equivalent ( $\mathrm{p}<0.001$ and $\mathrm{p}=0.005$, respectively), which suggests that the shipment of the control sample between the repositories and the IQAC did not negatively affect the sample integrity (data not shown).

Table 1

Stage 1 - PBMC initial viability, yield, and processing time at the site-affiliated laboratories.

\begin{tabular}{|c|c|c|c|}
\hline & $\begin{array}{l}\text { \% Viability } \\
\text { (Mean +/-SD) }\end{array}$ & $\begin{array}{l}\text { Cell yield } \\
\left(\times 10^{6} \text { cells } / \mathrm{mL} \text { of blood }\right) \\
(\text { Mean }+/-\mathrm{SD})\end{array}$ & $\begin{array}{l}\text { Processing time } \\
\text { (hours:min) } \\
\text { (Mean }+/-\mathrm{SD} \text { ) }\end{array}$ \\
\hline All site affiliated laboratories values & $98.3+/-1.5$ & $1.14+/-0.5$ & $4: 35+/-1: 23$ \\
\hline Acceptance criteria & $\geq 95$ & $0.7-3.0$ & $\leq 8: 00$ \\
\hline$\%$ of sites within acceptance criteria & 100 & 100 & 100 \\
\hline$\%$ of total specimens within acceptance criteria & 97.7 & 87.7 & 99.3 \\
\hline
\end{tabular}




\section{A}
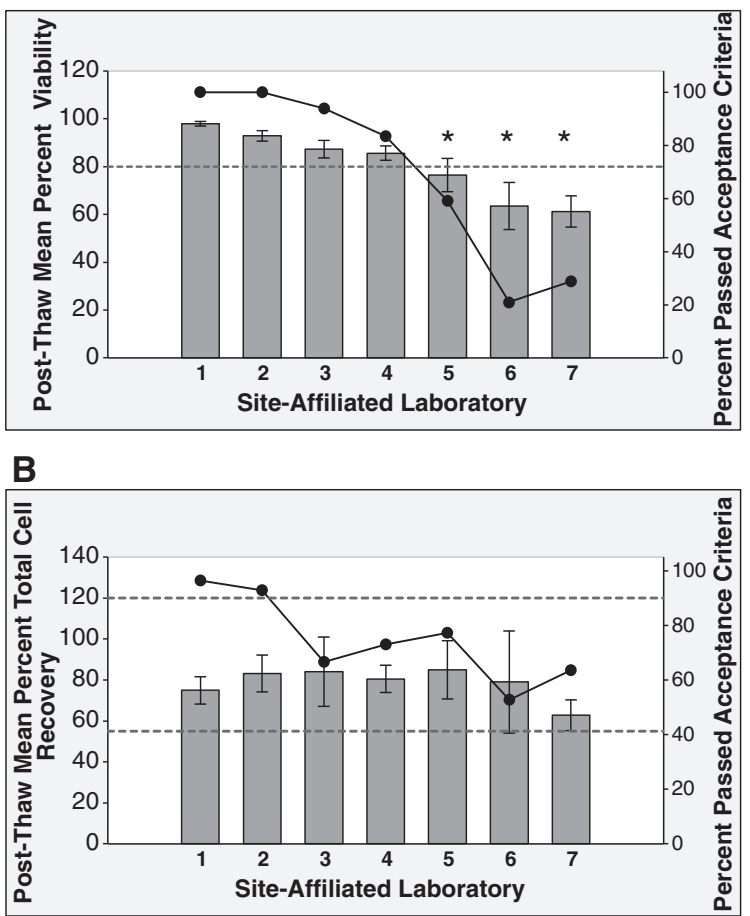

Fig. 2. Stage 2 - Cryopreserved PBMC viability and cell recovery after thawing at the repositories. The Day 1 PBMC post-thaw viability (Panel A) and total cell recovery (Panel $B$ ) QC results from PBMC preparations collected over five years from each site-affiliated laboratory are presented. The bars represent the raw data mean $+/-95 \%$ confidence interval $(\mathrm{CI})$ for the $\mathrm{QC}$ results (left axis). The circle markers on the line represent the percent of the PBMC preparations from each site-affiliated laboratory that passed the acceptance criteria (right axis). The asterisks in Panel A indicate that the viability results differed between site-affiliated laboratories based on non-overlapping model-based $95 \% \mathrm{CI}$ boundaries of the means. The upper and lower limits of the acceptance criteria are indicated by the horizontal dotted line(s) in each of the graphs. Acceptance criteria for PBMCs: post thaw viability $\geq 80 \%$; and post-thaw total cell recovery $55-120 \%$.

Control sample post-thaw QC data collected over two years are presented in Fig. 3 for each thawing location. One hundred percent of all the control sample vials exhibited post-thaw viability values that passed the acceptance criteria with a consensus mean viability $(+/-\mathrm{SD})$ of $97.4 \%+/-1.7 \%$. As shown in Fig. 3A, the mean control sample viability was within the acceptance criteria at each thawing location (95.9 to 99.0\%). There is no evidence that the control sample post-thaw viabilities at the four thawing locations are not equivalent (all p-values $<0.001$ ).

The control sample cell recovery results indicate that $99.0 \%$ of vials passed the acceptance criteria with a consensus mean recovery $(+/-\mathrm{SD})$ of $86.7 \%+/-11.9 \%$. The percentage of the vials that passed the acceptance criteria ranged from 92.6 to $100 \%$ depending on the thawing location (Fig. 3B). The mean control sample cell recovery at each thawing location was within the acceptance criteria and ranged from 78.6 to $97.5 \%$. Although there was statistical evidence that the recoveries at the four thawing locations were not equivalent, $99.0 \%$ of the control sample vials showed postthaw recovery within acceptance criteria (Fig. 3B).
The mean thawing quarterly results were plotted for each liquid nitrogen freezer (Fig. 4). A regression analysis was performed to determine whether the integrity of the control samples was maintained over time in storage. As shown in Fig. $4 \mathrm{~A}$, the mean post-thaw viability values from each repository freezer remained unchanged over the two-year period of monitoring. There is no statistical evidence that the post-thaw viability values for each of the repository freezers are trending downward over time (all p-values $<0.001$ ). As shown in Fig. 4B, the mean post-thaw cell recovery values from each repository freezer remained within the acceptance criteria over the two-year period of monitoring. However, there is statistical evidence that the post-thaw cell recovery values from repository 3 trended downward overtime $(\mathrm{p}$-value $=1.00$, otherwise all $\mathrm{p}$-values $<0.001)$.

\section{Discussion}

In this study, we present results obtained from a PBMC QA program designed to assess PBMC integrity upon initial isolation at the international CHAVI site-affiliated laboratories (Stage 1), after cryopreservation, handling and shipment to the CHAVI repositories (Stage 2) and upon long-term storage in liquid nitrogen freezers at the CHAVI repositories (Stage 3). Acceptance
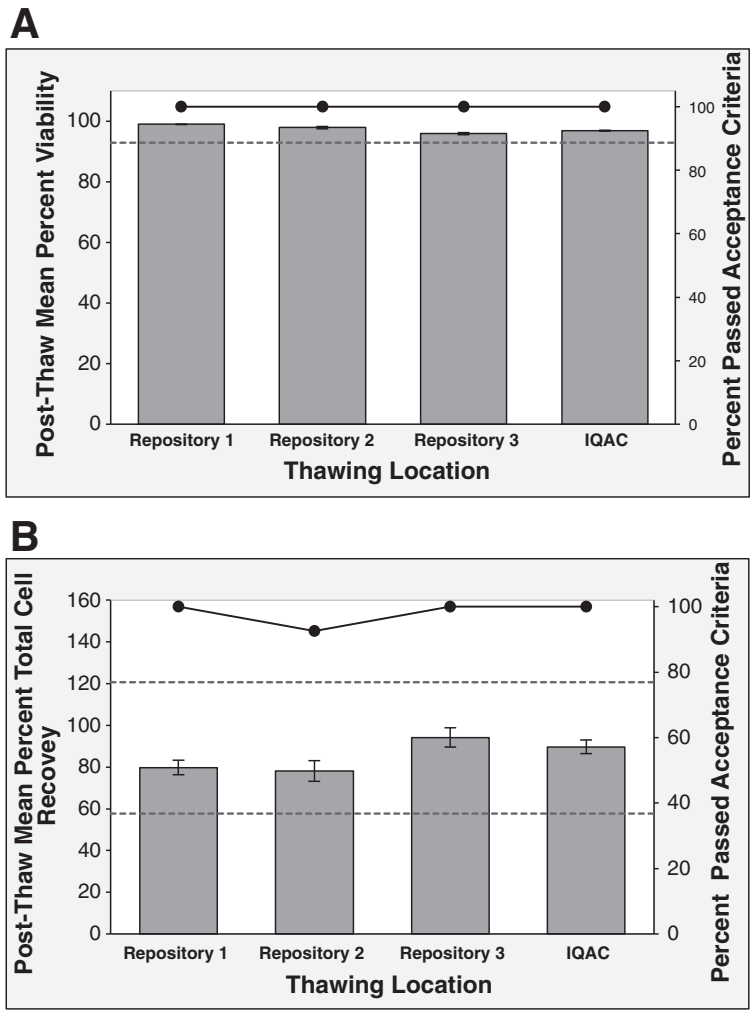

Fig. 3. Stage 3 - Sentinel Program for long-term storage of cryopreserved PBMCs at the repositories. The control sample post-thaw viability (Panel A) and Day 1 total cell recovery (Panel $B$ ) QC results collected over two years from each thawing location are presented. The bars represent the raw data mean $+/-95 \% \mathrm{CI}$ for the viability results and the model-based mean $+/-95 \% \mathrm{CI}$ for the recovery results (left axis). The circle markers on the line represent the percent of the control sample vials from each thaw site that passed the acceptance criteria (right axis). Acceptance criteria for the control samples: post-thaw viability $\geq 92.9 \%$; post-thaw recovery $57.7-120.7 \%$. 


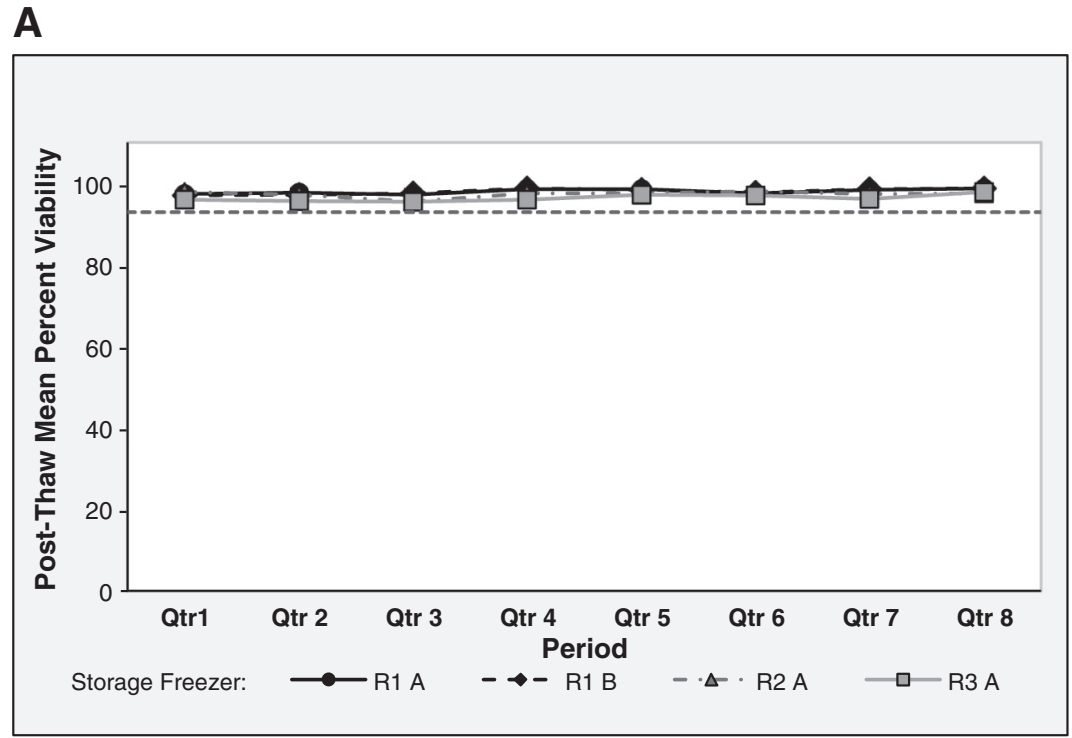

B

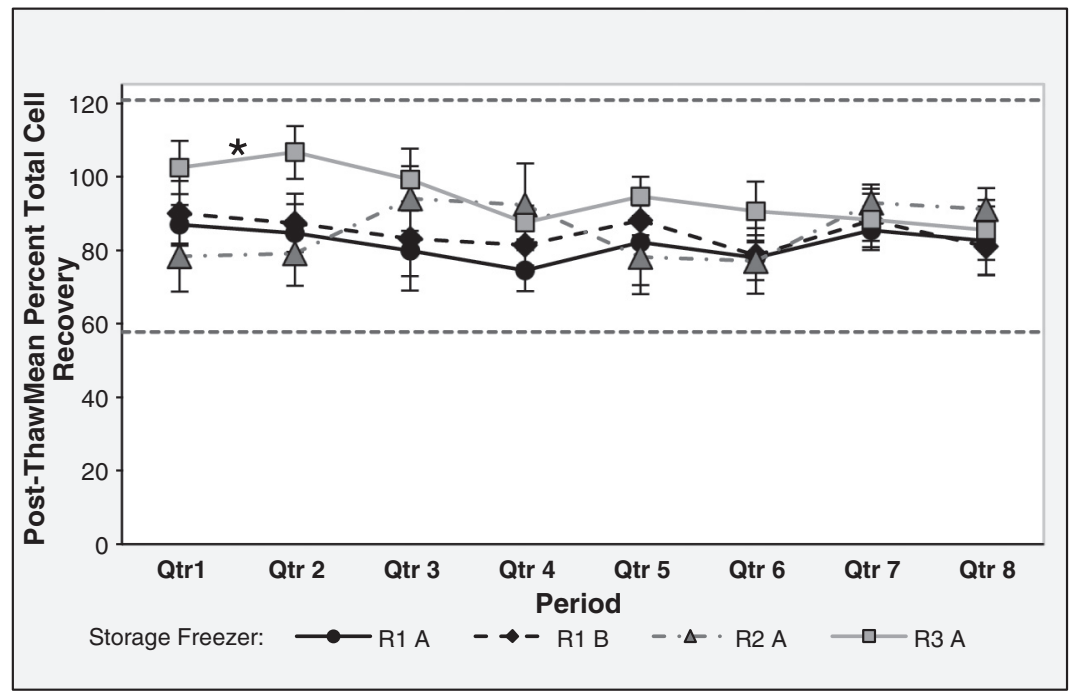

Fig. 4. Stage 3 - Sentinel Program for long-term storage of cryopreserved PBMCs at the repositories: analysis of each repository freezer over time. Post-thaw viability (Panel $A$ ) and Day 1 total cell recovery (Panel $B)$ QC results from the control sample vials stored in each repository freezer [Freezers A and B at the central repository $1(R 1 A, R 1 B)$; Freezer $A$ at the central repository $2(R 2 A)$; and Freezer $A$ at the repository $3(R 3 A)$ ] over two years are presented. The markers represent the raw data mean $+/-95 \% \mathrm{CI}$ for the QC results. The asterisk in Panel B indicates that, by regression analysis, Freezer A at repository 3 control sample post-thaw recoveries changed over time $(\mathrm{p}=1.000)$. The upper and lower limits of the acceptance criteria are indicated by the horizontal dotted line(s) in each of the graphs. Acceptance criteria for the control samples: post-thaw viability $\geq 92.9 \%$; and post-thaw recovery $57.7-120.7 \%$.

criteria were set for each key QC indicator and corrective actions, including site visits and necessary retraining, were implemented in the event of QC failures. It is important to note that the CHAVI PBMC QA program operated in site-affiliated laboratories and repositories that followed GCLP, including the use of CHAVI Central SOPs, training and competency assessments prior to initiating the study, and the monitoring and maintenance of the facility and equipment.

The integrity of the PBMCs upon isolation appeared high since the mean PBMC initial viability, PBMC yield and processing time at each site-affiliated laboratory were within the acceptance criteria (Table 1). 97.7\% of PBMC preparations had initial viabilities of $\geq 95 \%, 87.7 \%$ had yields of 0.7 $3.0 \times 10^{6} \mathrm{PBMC} / \mathrm{mL}$ blood, and $99.3 \%$ of the PBMCs were frozen within $8 \mathrm{~h}$ of blood collection. PBMC viability values were consistently high at five site-affiliated laboratories, the PBMC yields were within the acceptance criteria at each site-affiliated laboratory and, with the exception of one site, were similar across site-affiliated laboratories and over time. One site-affiliated laboratory exhibited higher PBMC yields at all times. Since this site-affiliated laboratory used the Vi-Cell instrument without RBC lysis to count cells, counting of RBCs 
may have contributed to the high PBMC yield values. Subsequent decreases in yield occurred after the site-affiliated laboratory began to verify any out of criteria yield results obtained with the Vi-Cell automated counter, by using hemacytometer counts.

Corrective action instituted by the site-affiliated laboratory managers when PBMC QC values did not meet the acceptance criteria included observation and retraining of the PBMC processing operators. Common recommendations included decreasing the force used and the formation of bubbles during pipetting and cell resuspension and the verification of out of criteria results. Additional recommendations for the improvement of PBMC yield included ensuring that the centrifugation speeds and brake settings were correct; that the PBMC layer was completely and cleanly harvested without excess plasma; and that the hemacytometer and cover glass were clean and free of scratches. In the few instances where PBMC processing times were greater than $8 \mathrm{~h}$, the root cause was usually a delay in the transport of the blood specimens from the clinic to the siteaffiliated laboratory, and corrective actions were instituted to decrease this delay. This suggests that the continuous monitoring of the PBMC QC coupled with observation and retraining, where necessary, provided an important feedback mechanism to reach and maintain high initial PBMC integrity.

Since many cell-mediated-immunity assays using cryopreserved PBMCs are dependent on the post-thaw PBMC viability (Cox et al., 2005; Kreher et al., 2003; Reimann et al., 2000; Sleasman et al., 1997; Weinberg et al., 2000, 2009, 2010), the results from the Stage 2 highlight how important it is to monitor post-thaw viability of cryopreserved PBMC. Earlier studies suggested that short-term storage of PBMC in liquid nitrogen at the site-affiliated laboratories and shipment in liquid nitrogen to the repositories results in higher PBMC post-thaw viability and recovery in comparison to short-term storage at $-70{ }^{\circ} \mathrm{C}$ and shipment on dry ice (Bull et al., 2007). Therefore, the decision was made to use liquid nitrogen for short-term site-affiliated laboratory storage and for shipment to the repositories. However, it should be noted that, in resource poor settings, careful consideration must be given to the maintenance of reliable supplies of liquid nitrogen, including the establishment of on-site liquid nitrogen production facilities where necessary. In fact, the two site-affiliated laboratories with the lowest PBMC post-thaw viability QC values had a persistent problem maintaining adequate liquid nitrogen.

The sentinel control sample post-thaw QC results from the three CHAVI repositories and the IQAC, which was utilized as a CHAVI-independent third party, were used to identify potential problems in the long-term storage of PBMC specimens. The thawing of sentinel control samples was used to monitor the freezer storage conditions without the need to thaw CHAVI cryopreserved PBMCs, and it also provided a direct comparison between post-thaw viability and recovery results obtained from the four thawing locations. This was especially important since the central repositories thawed PBMCs from the site-affiliated laboratories to monitor the quality of the PBMCs produced for CHAVI (Stage 2) and to determine the competency of the site-affiliated laboratory operators to isolate PBMCs.

Corrective action instituted by the site-affiliated laboratory managers when PBMC post-thaw viability and cell recovery did not meet the acceptance criteria included observation and retraining of the PBMC processing operators and those involved in PBMC shipments. Additional training by the central repositories was also instituted at site-affiliated laboratories 5, 6, and 7 . The common recommendations for the improvement of initial PBMC viability and yield also held for the improvement of PBMC post-thaw QC values. These included ensuring that the residual medium remaining after the final wash prior to the addition of the cryopreservation solution was adequately removed and that the chilled cryopreservation solution was added drop-wise to the chilled cell suspension. A consistent problem identified at site-affiliated laboratories 6 and 7 was a difficulty in maintaining adequate supplies of liquid nitrogen, with possible failure to maintain the cryopreserved PBMC specimens at cryogenic temperatures in liquid nitrogen vapor/transfer pans during inventory procedures or preparation for shipment.

There was no evidence that the acute HIV +, established $\mathrm{HIV}+$ and HIV negative cohort PBMC initial viability, yield and processing time were not equivalent using the predefined equivalence margins. A statistical analysis of the effect of HIVstatus on PBMC post-thaw viability and recovery was not performed because samples from established HIV-infected participants were available for thawing only from one site-affiliated laboratory, and the samples thawed from the other site-affiliated laboratories were almost exclusively from HIV-negative participants. However, comparable post-thaw recoveries of viable PBMC from HIV + and HIV negative cohorts were obtained in two multicenter study (Kleeberger et al., 1999; Weinberg et al., 2009; Aziz et al, 2013), supporting the validity of using either cohort PBMC to monitor these QC indicators, as done in the studies described herein.

Ultimately, the quality-controlled CHAVI PBMC specimens were successfully utilized in numerous immunogenicity studies, requiring viable and functional cells, by international CHAVI end user laboratories. Supplementary Table S1 lists 21 selected publications within 2013, on functional immunogenicity studies performed using CHAVI PBMC, which were processed, cryopreserved, shipped and stored as part of the CHAVI PBMC QA oversight program described in this manuscript. Supplementary Figs. S2 and S3 clearly depict the detection of low frequency, functional antigen-specific $\mathrm{T}$ cells obtained from the thawed PBMC stored at CHAVI central repository 1, providing evidence that the integrity of the CHAVI specimens, assessed by the CHAVI QA Program, was indeed maintained.

The quarterly Stage 3 results presented in Fig. 4 suggest that the viability and recovery of the cryopreserved PBMCs stored in CHAVI repositories liquid nitrogen freezers were maintained long-term. Our study has only examined the post-thaw viability and recovery of the sentinel cryopreserved control samples over two years. Further studies are ongoing to monitor these key quality indicators for control samples over longer lengths of storage time, as described by other multicenter studies (Kleeberger et al., 1999).

The yearly costs of conducting the comprehensive CHAVI PBMC QA oversight program, which included all QC activities at the site-affiliated laboratories, at the repositories and IQAC for all stages described herein, were estimated to be approximately \$3000/year/site-affiliated laboratory. This cost estimate was calculated in fiscal year 2013, and it is provided to aid future consortia in the planning of similar comprehensive PBMC QA programs.

Each of the stages of the CHAVI PBMC QA oversight program monitored PBMC quality indicators critical for the 
successful use of cryopreserved PBMC in cell-mediatedimmunity assays. The results suggest that it is more difficult to reach the post-thaw viability of $\geq 80 \%$ acceptance criteria (Stage 2) than to achieve the acceptance criteria for PBMC initial viability, yield, processing time (Stage 1 ) or for postthaw recovery (Stage 2 ). In this sense, the most cost-effective portion of the CHAVI PBMC QA Program has been the Stage $2-$ Cryopreserved $P B M C$ viability and cell recovery after thawing at the repositories because it effectively identified site-affiliated laboratories requiring additional training or resources. The Stage 3 Sentinel Program for long-term storage of cryopreserved PBMCs at the repositories assured that PBMC integrity was maintained during storage and monitored the comparability of PBMC thawing and counting at the repositories.

\section{Conclusions}

The CHAVI PBMC QA oversight program assessed the integrity of PBMC obtained from multiple international sites at isolation and after subsequent cryopreservation, handling, shipment, thaw and storage at the repositories. All of the nine site-affiliated laboratories exhibited high PBMC QC performance for Stage 1 and isolated PBMC with high initial viability, expected PBMC yields and processing times of $\leq 8 \mathrm{~h}$. The Stage 2 assessment at the repositories resulted in the ability to identify site-affiliated laboratories that required additional training or resources for the production of high quality cryopreserved PBMCs having a viability of greater than $80 \%$ upon thaw. The CHAVI PBMC QA oversight program also assessed that stable conditions were monitored and maintained during long-term storage at the repositories.

Overall, the CHAVI PBMC QA oversight program results highlight the relative importance of each of its stages to the ultimate goal of preserving specimen integrity from collection to long-term storage. The most crucial results were derived from Stage 2, where the quality of PBMC was tested by thawing at the central repositories, although all parts of the program contributed substantially to monitoring specimen integrity. To help the design of similar future programs, we estimated that the cost per year required to support this program was approximately $\$ 3000 /$ year/site-affiliated laboratory. The CHAVI PBMC QA Program is one of the several parallel programs utilized by international HIV-network organizations to control specimen integrity. Lessons learned from each of these programs may aid in the design of a unified one in the future.

\section{Abbreviations}

CHAVI Center for HIV/AIDS Vaccine Immunology

CI confidence interval

GCLP Good Clinical Laboratory Practice

HVTN HIV Vaccine Trials Network

FBS fetal bovine serum

IQAC Immunology Quality Assessment Center

LDMS Laboratory Data Management System

NICD National Institute for Communicable Diseases

PBMC Peripheral Blood Mononuclear Cell

PCA Personal Cell Analysis

PTID Participant Identification Number

QA quality assurance
SD standard deviation

SOP standard operating procedure

UNC University of North Carolina at Chapel Hill

Supplementary data to this article can be found online at http://dx.doi.org/10.1016/j.jim.2014.05.013.

\section{Acknowledgments}

This research was supported by CHAVI grant AI067854 from the Division of AIDS, NIAID, NIH.

We thank the Laboratory and QA Managers of the CHAVI site-affiliated laboratories involved in this study: Brandi Davis, Hiroshi Mohri, Leslie St. Bernard (Aaron Diamond AIDS Research Center, New York, New York, USA); Itumeleng Kgotlagomang, Lindiwe Nlanqulela (Aurum Institute for Health Research, Klerksdorp, South Africa); Natasha Samsunder (CAPRISA, University of KwaZulu-Natal, Durban, South Africa); Kirthi Hira, Naazneen Moolla (CLS, National Health Laboratory Service/Wits Health Consortium of the University of the Witwatersrand, Johannesburg, South Africa); Ella Bernstein, (Duke University, Durham, North Carolina); Chifundo Chipungu, Deborah Kamwendo Demster, (JHUR Project, Malawi College of Medicine, Blantyre, Malawi); Caroline Chevallier, Ireen Kiwelu, Jean Gratz, John Gardner Gaddiel, Shanette Nixon (Kilimanjaro Christian Medical Centre, Moshi, Tanzania); Jennifer Serwanga (MRC/UVRI, Entebbe, Uganda); and Nicole Carpenetti, George Joaki, and Gerald Tegha (UNC Project, Lilongwe, Malawi). We thank the CHAVI Protocol study participants and the CHAVI Clinical Sites Teams, Clinical Core Team and Protocol Teams and Principal Investigators for their contributions.

We also thank all members of the CHAVI Site-Associated Laboratories; David Mokgokolo, Greg Khoury, Vinodh Edward and the Laboratory Oversight Group; Ada Cachafeiro, Charles West, Daniel Wilkinson, Debra de Assis Rosa, Innocent Zgambo, MariAnne Jessup-Cumming, Michael Diago, Rachana Kshatriya, Thabisile Gwala, Tyler Hawkins and the CHAVI Repository Teams; Ambrosia Garcia, Eugene Urrutia and the IQAC; Darcy McMullin, Elizabeth Petzold, and the CHAVI Program Team; Ayana Moore, Carolyn Yanavich, Danielle Haley, Deborah Hilgenberg, Marybeth McCauley, Scott Rose and Family Health International; Marlene Cooper, Howard Gutzman and FSTRF; Sarah Ramsay, Shane Coultas and SCHARP; and Ana Sanchez for critically reviewing the manuscript.

\section{References}

Afonso, G., Scotto, M., Renand, A., Arvastsson, J., Vassilieff, D., Cilio, C.M., Mallone, R., 2010. Critical parameters in blood processing for T-cell assays: validation on ELISpot and tetramer platforms. J. Immunol. Methods 359, 28.

Aziz, N., Margolick, J.B., Detels, R., Rinaldo, C.R., Phair, J., Jamieson, B.D., Butch, A.W., 2013. Value of a quality assessment program in optimizing cryopreservation of peripheral blood mononuclear cells in a multicenter study. Clin. Vaccine Immunol. 20, 590.

Bull, M., Lee, D., Stucky, J., Chiu, Y.L., Rubin, A., Horton, H., McElrath, M.J., 2007. Defining blood processing parameters for optimal detection of cryopreserved antigen-specific responses for HIV vaccine trials. J. Immunol. Methods 322, 57

Cox, J.H., Ferrari, G., Kalams, S.A., Lopaczynski, W., Oden, N., D'Souza, M.P., E.C.S Group, 2005. Results of an ELISPOT proficiency panel conducted in 11 laboratories participating in international human immunodeficiency virus type 1 vaccine trials. AIDS Res. Hum. Retrovir. 21, 68. 
Disis, M.L., dela Rosa, C., Goodell, V., Kuan, L.Y., Chang, J.C.C., Kuus-Reichel, K. Clay, T.M., Lyerly, H.K., Bhatia, S., Ghanekar, S.A., Maino, V.C., Maecker, H.T. 2006. Maximizing the retention of antigen specific lymphocyte function after cryopreservation. J. Immunol. Methods 308, 13.

Ducar, C., Smith, D., Pinzon, C., Stirewalt, M., Cooper, C., McElrath, M.J., Hural, J., the NIAID HIV Vaccine Trials Network, 2014. Benefits of a comprehensive quality program for cryopreserved PBMC covering 28 clinical trials sites utilizing an integrated, analytical web-based portal. J. Immunol. Methods. http://dx.doi.org/10.1016/j.jim.2014.03.024 (Apr 4. pii: S0022-1759(14)00113-6, Epub ahead of print).

Dyer, W.B., Pett, S.L., Sullivan, J.S., Emery, S., Cooper, D.A., Kelleher, A.D. Lloyd, A., Lewin, S.R., 2007. Substantial improvements in performance indicators achieved in a peripheral blood mononuclear cell cryopreservation quality assurance program using single donor samples. Clin. Vaccine Immunol. 14, 52.

Ezzelle, J., Rodriguez-Chavez, I.R., Darden, J.M., Stirewalt, M., Kunwar, N., Hitchcock, R., Walter, T., D'Souza, M.P., 2008. Guidelines on good clinical laboratory practice: bridging operations between research and clinical research laboratories. J. Pharm. Biomed. Anal. 46, 18.

Garcia, A., Keinonen, S., Sanchez, A.M., Ferrari, G., Denny, T.M., Moody, M.A. 2014. Leukopak PBMC Sample Processing for Preparing Quality Control Material to Support Proficiency Testing Programs, (in this JIM issue).

Kierstead, L.S., Dubey, S., Meyer, B., Tobery, T.W., Mogg, R., Fernandez, V.R., Long, R., Guan, L., Gaunt, C., Collins, K., Sykes, K.J., Mehrotra, D.V., Chirmule, N., Shiver, J.W., Casimiro, D.R., 2007. Enhanced rates and magnitude of immune responses detected against an HIV vaccine: effect of using an optimized process for isolating PBMC. AIDS Res. Hum. Retrovir. 23, 86

Kleeberger, C.A., Lyles, R.H., Margolick, J.B., Rinaldo, C.R., Phair, J.P., Giorgi, J.V., 1999. Viability and recovery of peripheral blood mononuclear cells cryopreserved for up to 12 years in a multicenter study. Clin. Diagn. Lab. Immunol. 6, 14.

Kreher, C.R., Dittrich, M.T., Guerkov, R., Boehm, B.O., Tary-Lehmann, M., 2003. CD4 + and CD8 + cells in cryopreserved human PBMC maintain full functionality in cytokine ELISPOT assays. J. Immunol. Methods 278, 79.

Olemukan, R.E., Eller, L.A., Ouma, B.J., Etonu, B., Erima, S., Naluyima, P., Kyabaggu, D., Cox, J.H., Sandberg, J.K., Wabwire-Mangen, F., Michael, N.L., Robb, M.L., de Souza, M.S., Eller, M.A., 2010. Quality monitoring of HIV-1infected and uninfected peripheral blood mononuclear cell samples in a resource-limited setting. Clin. Vaccine Immunol. 17, 910.

Ramachandran, H., Laux, J., Moldovan, I., Caspell, R., Lehmann, P.V. Subramanian, R.A., 2012. Optimal thawing of cryopreserved peripheral blood mononuclear cells for use in high-throughput human immune monitoring studies. Cells 1,313 .

Reimann, K.A., Chernoff, M., Wilkening, C.L., Nickerson, C.E., Landay, A.L., ACTG Immunology Advanced Technology Laboratories, 2000. Preservation of lymphocyte immunophenotype and proliferative responses in cryopreserved peripheral blood mononuclear cells from human immunodeficiency virus type 1-infected donors: implications for multicenter clinical trials. Clin. Diagn. Lab. Immunol. 7, 352.
Riou, C., Ganusov, V.V., Campion, S., Mlotshwa, M., Liu, M.K., Whale, V.E., Goonetilleke, N., Borrow, P., Ferrari, G., Betts, M.R., Haynes, B.F., McMichael, A.J., Gray, C.M., 2012 Mar 1. Distinct kinetics of Gag-specific $\mathrm{CD} 4+$ and $\mathrm{CD} 8+\mathrm{T}$ cell responses during acute HIV-1 infection. J Immunol. 188 (5), 2198-2206. http://dx.doi.org/10.4049/jimmunol. 1102813 Epub 2012 Jan 27.

Sarzotti-Kelsoe, M., Cox, J., Cleland, N., Denny, T., Hural, J., Needham, L., Ozaki, D., Rodriguez-Chavez, I.R., Stevens, G., Stiles, T., Tarragona-Fiol, T., Simkins, A., 2009. Evaluation and recommendations on Good Clinical Laboratory Practice (GCLP) guidelines for phase I-III clinical trials. PLoS Med. 6 (5), e1000067. http://dx.doi.org/10.1371/journal.pmed.1000067.

Sleasman, J.W., Leon, B.H., Aleixo, L.F., Rojas, M., Goodenow, M.M., 1997. Immunomagnetic selection of purified monocyte and lymphocyte populations from peripheral blood mononuclear cells following cryopreservation. Clin. Diagn. Lab. Immunol. 4, 653.

Smith, J.G., Liu, X., Kaufhold, R.M., Clair, J., Caulfield, M.J., 2001. Development and validation of a gamma interferon ELISPOT assay for quantitation of cellular immune responses to varicella-zoster virus. Clin. Diagn. Lab. Immunol. 8, 871.

Smith, J.G., Joseph, H.R., Green, T., Field, J.A., Wooters, M., Kaufhold, R.M., Antonello, J., Caulfield, M.J., 2007. Establishing acceptance criteria for cell-mediated-immunity assays using frozen peripheral blood mononuclear cells stored under optimal and suboptimal conditions. Clin. Vaccine Immunol. 14, 527.

Stiles, T., Grant, V., Mawbey, N., 2003. Good clinical laboratory practice. A Quality System for Laboratories which undertake the Analyses of Samples from Clinical TrialsBritish Association of Research Quality Assurance, Ipswich, United Kingdom, p. 1.

Weinberg, A., Zhang, L., Brown, D., Erice, A., Polsky, B., Hirsch, M.S., Owens, S., Lamb, K., 2000. Viability and functional activity of cryopreserved mononuclear cells. Clin. Diagn. Lab. Immunol. 7, 714.

Weinberg, A., Louzao, R., Mussi-Pinhata, M.M., Cruz, M.L., Pinto, J.A., Huff, M.F., de Castro, A.C., Sucupira, M.C., Denny, T.N., 2007. Quality assurance program for peripheral blood mononuclear cell cryopreservation. Clin. Vaccine Immunol. $14,1242$.

Weinberg, A., Song, L.Y., Wilkening, C., Sevin, A., Blais, B., Louzao, R., Stein, D., Defechereux, P., Durand, D., Riedel, E., Raftery, N., Jesser, R., Brown, B., Keller, M.F., Dickover, R., McFarland, E., Fenton, T., Pediatric ACTG Cryopreservation Working Group, 2009. Optimization and limitations of use of cryopreserved peripheral blood mononuclear cells for functional and phenotypic T-cell characterization. Clin. Vaccine Immunol. $16,1176$.

Weinberg, A., Song, L.Y., Wilkening, C.L., Fenton, T., Hural, J., Louzao, R., Ferrari, G., Etter, P.E., Berrong, M., Canniff, J.D., Carter, D., De fawe, O.D., Garcia, A., Garrelts, T.L., Gelman, R., Lambrecht, L.K., Pahwa, S., PilakkaKanthikeel, S., Shugarts, D.L., Tustin, N.B., 2010. Optimization of storage and shipment of cryopreserved peripheral blood mononuclear cells from HIV-infected and uninfected individuals for ELISPOT assays. J. Immunol. Methods 363, 42 